\title{
LUTTE CONTRE LES PARASITES CHEZ LE KARITE
}

\author{
D. SORO ${ }^{1}$, A. N'DA-ADOPO ${ }^{2}$, K. P. DA ${ }^{1}$ et D. TRAORE ${ }^{1}$ \\ ${ }^{1}$ Laboratoire de Botanique, UFR Biosciences, Université de Cocody. \\ 22 BP 582 Abidjan 22, Côte d'Ivoire \\ ${ }^{2}$ Centre National de Recherche agronomique, Direction Régionale Nord. \\ BP 856 Korhogo, Côte d'Ivoire.
}

\begin{abstract}
RESUME
Le karité, espèce typique des savanes soudaniennes d'Afrique, joue un rôle important dans le développement économique des pays producteurs, grâce à la commercialisation des amandes dont on extrait le beurre de karité. Ce beurre est utilisé dans la fabrication de nombreux produits, notamment dans l'alimentation locale, la chocolaterie, la cosmétique et la pharmacopée. Toutefois, les peuplements de karités font de plus en plus l'objet d'attaques sévères par les Loranthaceae, plantes vasculaires parasites communément appelées guis. Une étude de la lutte contre ces parasites a été effectuée sur le parc naturel à karité de Tengrela, au Nord de la Côte d'Ivoire. Les résultats acquis au cours de ces travaux de recherche, montrent que les attaques des Loranthaceae sont importantes sur le karité. Le déparasitage par émondage est un moyen de lutte que nous avons trouvé efficace contre les Loranthaceae, car, il coûte peu cher et est à la portée de la bourse du paysan. De plus, les karités correctement émondés régénèrent tous, rapidement, et produisent des fruits, environ 2 ans après.
\end{abstract}

Mots clés : Karité, Loranthaceae, parasite, émondage, Côte d'Ivoire.

\author{
ABSTRACT \\ STRUGGLE AGAINST PARASITES OF SHEA TREES
}

Shea tree, species typical of Africa's soudanian savannah, plays an important rôle in the economic development of the producer countries, thanks to the marketing of the almonds from which shea butter is extracted. This butter is used in many products, such as local food, chocolate, cosmetic and pharmacopoeia. Nevertheless, shea orchards are more and more subject to severe attacks by Loranthaceae, a vascular parasite plants, commonly called guis. Therefore, a pest management study was carried out on Tengrela's natural shea tree park, in northern Côte d'lvoire. The results show that Loranthaceae attacks are important on shea trees. The deparasitage by pruning as a control measure was found cost effective against Loranthaceae, with respect to farmers. Moreover, when properly pruned, all shea trees quickly regenerate and are productive about 2 years later.

Key words : Shea tree, Loranthaceae, parasite, pruning, Côte d'lvoire.

\section{INTRODUCTION}

Les plantes vasculaires de la famille des Loranthaceae parasitent de nombreuses espèces ligneuses. L'implantation de ces plantes sur les branches d'un sujet, provoque une diminution de la croissance de l'individu infesté, et l'hôte parasité devient économiquement inutilisable (Delabraze et al., 1972 ; Frochot et al., 1978). Toutefois, les Loranthaceae ne parasitent pas tous les arbres avec la même fréquence. Leur développement est largement conditionné par la nature de l'hôte. Le karité Vitellaria paradoxa C. F. Gaertn. (Sapotaceae) est l'un des hôtes les plus sensibles aux Loranthaceae. Ces parasites sans lui être spécifiques, ont trouvé en lui un hôte favorable et s'y fixe parfois en nombre considérable. Très rares sont les karités adultes non infestés par les Loranthaceae. 
Pourtant, le karité présente beaucoup d'intérêt pour les pays qui possèdent des peuplements conséquents de cette espèce. En effet, le beurre extrait de l'amande de karité, connaît de nombreuses utilisations. Il est utilisé par l'industrie alimentaire, cosmétique et pharmaceutique ainsi qu'en pharmacopée traditionnelle africaine. Le karité bénéficie ainsi d'un regain d'intérêt économique sur le plan international. II est alors nécessaire de connaître les parasites vasculaires en pleine expansion sur cet arbre, afin d'élaborer des méthodes de lutte efficaces contre ce fléau. Les aspects de la biologie et des mécanismes physiologiques par lesquels les Loranthaceae se fixent sur leurs hôtes et puisent leurs nutriments, ont été décrits par plusieurs auteurs tel que Salle (1977), Boussim (1991), Boussim et al., (1993), Traoré et al., (1996).

Initiée par l'Association Ivoirienne des Sciences Agronomique (A.I.S.A.) en 1994, suivie par l'Université de Cocody en 1996 et depuis 2000 par le Centre National de Recherche Agronomique (C.N.R.A), notre étude porte sur le parc naturel à karités de Tengrela (figure 1), dans le Nord de la Côte d'Ivoire. Il est situé au nord-est de la ville de Tengrela, en direction du village de Tamania, entre $10^{\circ} 29 \mathrm{~N}$ et $6^{\circ} 24 \mathrm{~W}$. Le climat de la région est du type tropical sec, avec deux principales saisons très opposées:

- une saison sèche, de novembre à avril, très marquée par l'harmattan entre décembre et janvier, avec des pointes de chaleur en février et mars ;
- une saison de pluies, de mai à octobre, avec des pluviométries maximales en août.

Les températures moyennes mensuelles varient entre $24^{\circ} \mathrm{C}$ et $33^{\circ} \mathrm{C}$. La région est essentiellement couverte par une formation végétale de type savane arborée.

Ce travail vise à présenter l'ampleur des attaques et les dégâts causés par les Loranthaceae sur le karité et proposer une méthode simple de lutte facilement applicable contre ces parasites.

\section{MATERIEL ET METHODES}

Le karité est un arbre à croissance lente, donc difficile à reproduire. Son délai de production est de 17 à 20 ans. Aussi, nos analyses portentelles sur un peuplement naturel : le parc naturel à karités de Tengrela. Ce parc est caractérisé par une formation homogène de karité. Selon Traoré et al., (1997), l'origine de ce parc serait liée au passage des sofas de SAMORY TOURE (grand chef guerrier musulman ayant résisté à la colonisation française) dans le Nord de la Côte d'Ivoire, vers 1898. Ces guerriers qui ont campé à cet endroit, lors du siège du village de Tengrela, auraient consommé la pulpe des fruits de karité et auraient abandonné une quantité importante de noix sur place. Les graines auraient germé en abondance. Le milieu a été éclairci par la suite pour rendre possible les cultures vivrières de sorgho, d'arachides et plus tard, industrielles

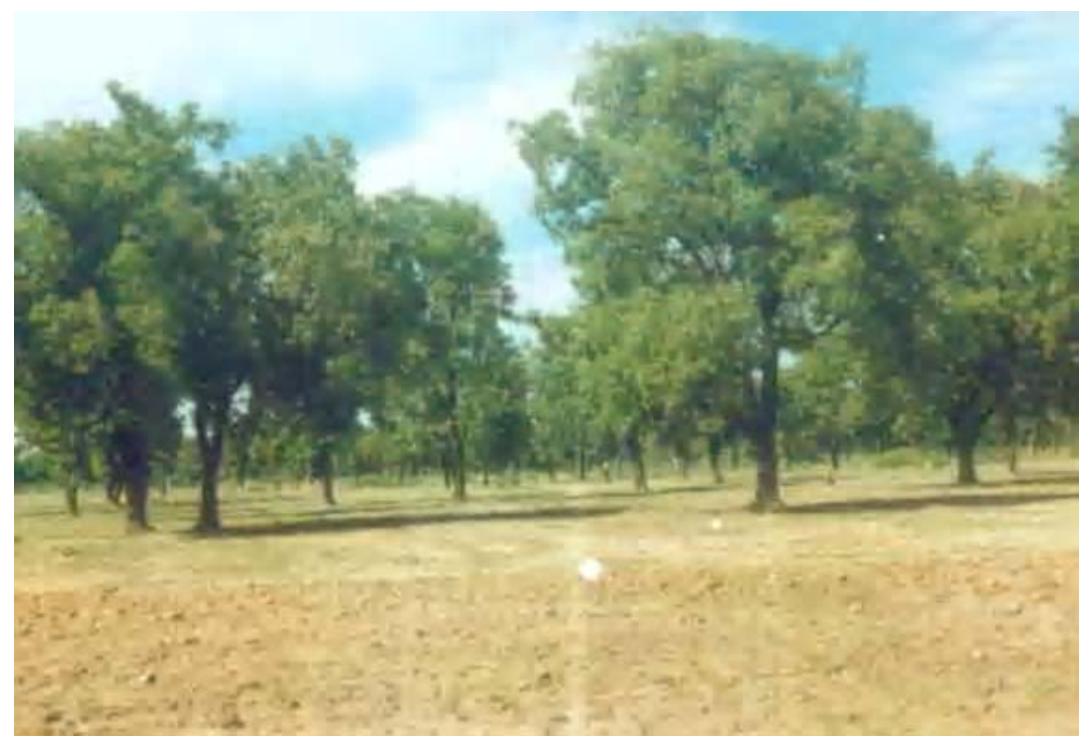

Figure 1 : Vue partielle du parc naturel à karités de Tengrela (Photo TRAORE 1997)

Partial View of Tengrela's natural park of shea trees 
de cotonniers. Le parc aurait donc plus de 100 ans d'âge. Le travail en peuplement naturel, pose le problème de répétition du nombre d'individus par échantillon (parcelle de 1 hectare).

Pour mener à bien notre étude, le parc a été quadrillé en 36 parcelles. Les parcelles numérotées de 1 à 17 , considérées comme entières ont une superficie de un hectare chacune ; les autres (parcelle 18 à 36) couvrent, chacune, moins d'un hectare. Afin de retrouver facilement la position des arbres dans le parc, les karités ont été marqués à la peinture à huile. Le système de numérotation utilisé est un couple de nombres séparés par un tiret ; le premier indiquant le numéro de la parcelle, le second, celui de l'arbre.

Les parcelles 1, 2, 5, 6, 13 et 14 ont été choisies suivant un plan en damier ou dispositif à témoin adjacent (figure 2), pour mener l'étude. Le traitement a consisté à émonder les arbres. Une personne monte à un arbre et examine les branches, les unes après les autres. Les branches sur lesquelles l'on rencontre au moins un parasite, sont sectionnées. La section de la branche parasitée doit se faire à environ $30 \mathrm{~cm}$ en amont du point de fixation du parasite, afin d'éliminer la totalité du système endophytique de ce dernier, sinon il régénère très rapidement de nouvelles tiges feuillées. Ici, toutes les branches des arbres ont été sectionnées car, chacune d'elles portait au moins un parasite. Les branches coupées ont été examinées non seulement pour identifier les espèces parasites, mais aussi pour déterminer le degré du parasitisme (nombre de parasites par arbre). Les arbres des parcelles 2, 6 et 14 ont été émondés entre mi-novembre et mi-décembre 1997, ceux des parcelles 1, 5 et 13 ont été laissés avec leurs parasites et constituent les témoins. Plus tard, ayant constaté que l'ampleur des attaques parasitaires varie avec la localisation des parcelles, nous avons émondé les arbres de la parcelle 17, entre mi-novembre et mi-décembre 1998, pour pouvoir comparer le degré du parasitisme de deux parcelles de bordure avec celui des parcelles 6 et 14, situées au milieu du parc.

Ayant constaté, après émondage, l'installation des parasites sur les jeunes rameaux, cette nouvelle infestation a été suivie de 1998 à 2001. Ces observations ont consisté à dénombrer les parasites qui s'implantent à nouveau sur les arbres émondés. La dynamique d'évolution des parasites sur les arbres émondés est comparée à celle des arbres non émondés qui constituent les témoins.

L'évolution de la fructification à partir des nouveaux rameaux a été également observée pendant la même période (de 1998 à 2001). Les fruits d'un arbre de karité n'ayant pas un développement synchrone, la production a été déterminée après collecte quotidienne des fruits tombés. Pour avoir la production annuelle par arbre, nous avons fait la somme des lots quotidiens.

Pour apprécier l'effet des parasites sur la production en fruits du karité, le degré du parasitisme et la production en fruits des arbres non émondés (arbres des parcelles 1, 5 et 13) ont été relevés au cours de la période 1998 à 2001. La production en fruits des arbres émondés est aussi comparée à celle des arbres non émondés.

Les analyses statistiques ont été réalisées avec le logiciel Excel, version 97 \& 5. 0/95 de Microsoft.

\section{RESULTATS}

Les résultats relatifs à l'ampleur des attaques des parasites sur le karité, montrent que les arbres du parc à karités de Tengrela sont fortement parasités (figure 3) par les Loranthaceae. En effet, au niveau des parcelles 2,6 et 14 dont les arbres ont été émondés en 1997 , nous avons trouvé seulement 3 karités sans parasites sur un total de 91 arbres traités. Aussi, environ $96 \%$ des karités adultes sont-ils attaqués par ces parasites vasculaires. Le degré de parasitisme enregistré est de 21 parasites en moyenne par arbre, avec un coefficient de variation de 89,14. Le coefficient de variation, très élevé, indique une forte variabilité du dégré du parasitisme d'un karité à l'autre. Les espèces de Loranthaceae récoltées sur le parc sont Agelanthus dodoneifolius (DC.) Polh et Wiens et Tapinanthus bangwensis (Engl. et Krause) Danser. Ces parasites se situent le plus souvent à la périphérie du houppier de leurs hôtes.

Le tableau 1 montre que l'importance des attaques des Loranthaceae sur le karité varie suivant les parcelles. Les parcelles 2 et 17 , situées en bordure du parc, présentent un degré de parasitisme élevé, avec respectivement 35,1 et 40,6 parasites par arbre. Les parcelles 6 et 14 , situées au milieu du parc, ont un niveau de 
parasitisme peu élevé, avec respectivement 19,6 et 20,5 parasites par arbre. Les coefficients de variation, élevés, révèlent une forte variabilité du degré de parasitisme d'un arbre à l'autre.

Les dégâts causés par les Loranthaceae sur le karité sont multiples. Ces parasites provoquent toujours des déformations au niveau des points de fixation sur leur hôte. On observe très souvent des cas de mortalité de parasites sur leur hôte. La dégénérescence des tissus des parasites morts, laisse une cavité dans l'hôte. Ces cavités laissées par les parasites dans l'hôte, provoquent une altération de la qualité du bois de celui-ci et le rendent plus vulnérable à d'autres

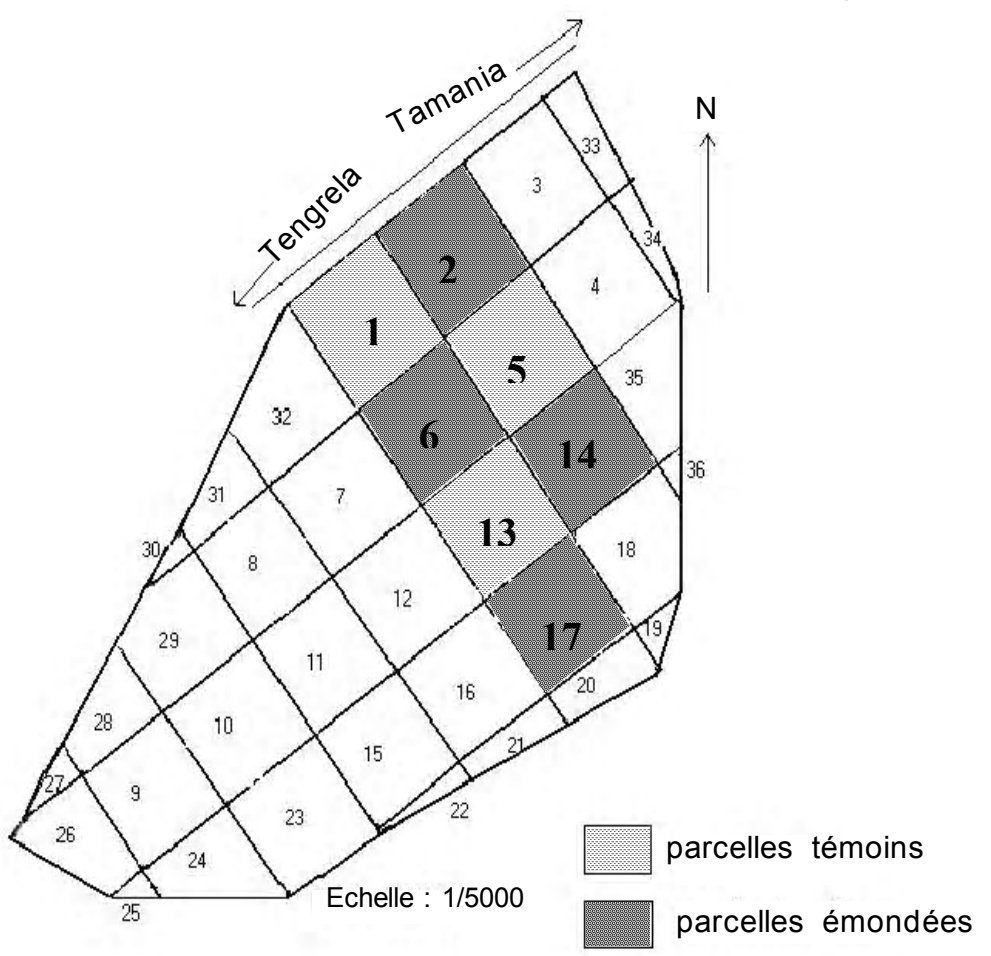

Figure 2 : Plan du parc naturel à karités de Tengrela

Plan of Tengrela's natural park of shea trees

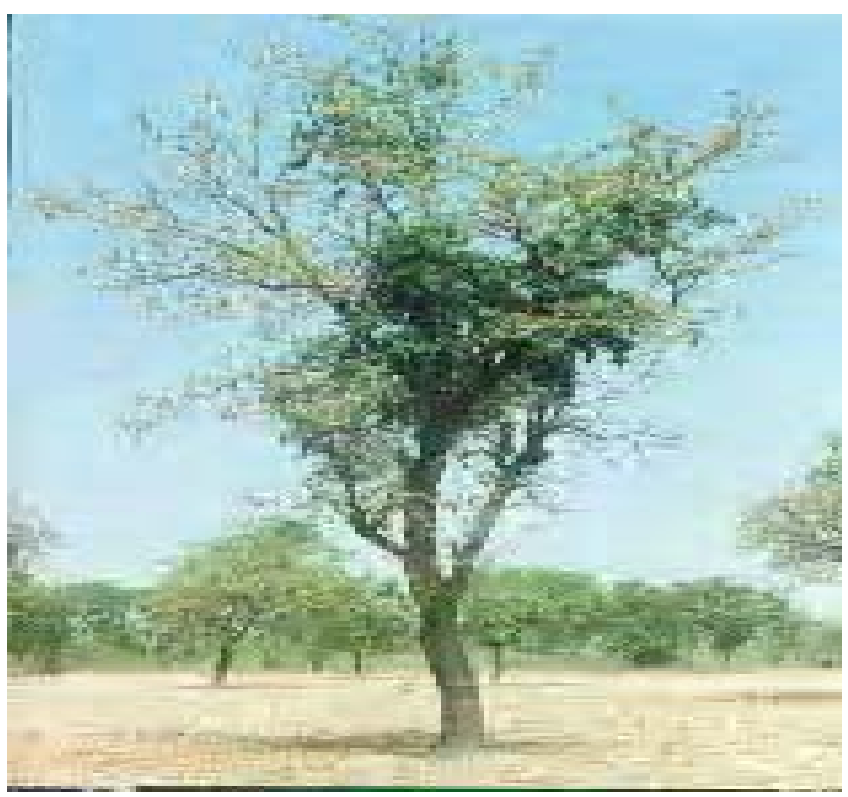

Figure 3 : Arbre de karité densément parasité (parasites en gris foncé) Photo TRAORE 1997

Densely parasited shea tree (parasites are grey deep) 
Tableau 1 : Degré de parasitisme des karités des parcelles 2, 6, 14 et 17

Degree of parasitism of shea trees of plots of land 2, 6, 14 and 17

\begin{tabular}{cccc}
\hline & \multicolumn{3}{c}{ Valeurs déterminées } \\
\cline { 2 - 4 } Parcelles émondées & $\begin{array}{c}\text { Nombre de karités } \\
(\text { arbre })\end{array}$ & $\begin{array}{c}\text { Moyenne du nombre } \\
\text { de parasites/arbre } \\
\text { (degré de parasitisme) }\end{array}$ & $\begin{array}{c}\text { Coefficient de } \\
\text { variation (\%) }\end{array}$ \\
\hline 2 & 26 & 35,1 & 65,5 \\
6 & 37 & 19,6 & 62,04 \\
14 & 28 & 20,5 & 99,6 \\
17 & 18 & 40,6 & 46,1 \\
moyenne & 27,5 & 28,95 & 68,31 \\
\hline
\end{tabular}

agents pathogènes. Les Loranthaceae provoquent donc d'importants dégâts technologiques du bois chez l'arbre hôte. Au niveau de la production en fruits, le tableau 2 montre de faibles corrélations négatives entre le niveau d'attaque parasitaire et la fructification du karité ; les arbres très parasités seraient en général moins productifs.
Les coefficients de variation, très élevés, auxquels nous parvenons, indiquent bien une forte variabilité de la production suivant les arbres. Toutefois les arbres émondés subissent une nouvelle infestation comme l'indique le tableau 4, avec notamment l'apparition, à partir de 1999, de points d'attaques parasitaires sur les arbres traités en 1997. les dégrés parasitaires

Tableau 2 : Corrélations entre le degré de parasitisme et la production en fruits des karités dans le parc à karité de Tengrela

Correlations between the degree of parasitism and production out of fruits of shea trees of Tengrela's park of shea trees

\begin{tabular}{lcccc}
\hline \multirow{2}{*}{ Paramètres } & \multicolumn{4}{c}{ Années d'observation } \\
\cline { 2 - 5 } & 1998 & 1999 & 2000 & 2001 \\
\hline Nombre de karités & 126 & 126 & 126 & 124 \\
Production totale (nombre de fruits) & 208897 & 178081 & 100097 & 139332 \\
Coefficient de corrélation & $-0,12$ & $-0,19$ & $-0,05$ & $-0,17$ \\
\hline
\end{tabular}

Les karités traités (figure 4) régénèrent rapidement et leur production fruitière s'établit 2 ans après émondage. En effet, les karités émondés en 1997 n'ont commencé à donner des fruits qu'à partir de 1999. Les productions moyennes enregistrées de 1999 à 2001, bien que faibles, connaissent une amélioration notable au fil des années (tableau 3). La reprise de la production en fruits est donc progressive et certains individus comme le 2-12, le 6-11 et le 6-26 produisent déjà près de 500 fruits chacun. observés de 1999 à 2001 sur les jeunes rameaux, sont faibles (tableau 4). Les coefficients de variation, très élevés $(1050,270,270,83)$, indiquent bien une forte variabilité du dégré du parasitisme sur les karités traités. Cette variabilité du degré du parasitisme serait-elle liée à une différence de résistance des karités à la contamination ? Les études ultérieures permettront certainement de répondre à cette question. 
Tableau 3 : Statistiques élémentaires de la production en fruits des karités après émondage en 1997, dans le parc à karités de Tengrela

Elementary Statistics of production out of fruits of shea trees, after pruning in 1997, in Tengrela's park of shea

\begin{tabular}{ccccc}
\hline \multirow{2}{*}{ Année } & \multicolumn{4}{c}{ Statistique élémentaire } \\
\cline { 2 - 5 } & Nombre de karités & $\begin{array}{c}\text { Moyenne de production } \\
\text { (nombre de fruits) }\end{array}$ & $\begin{array}{c}\text { Ecart type } \\
\text { (nombre de fruits) }\end{array}$ & $\begin{array}{c}\text { Coefficient de } \\
\text { variation (\%) }\end{array}$ \\
\hline 1998 & 91 & 0 & 0 & 0 \\
1999 & 91 & 15 & 51,67 & 344,46 \\
2000 & 91 & 17 & 54,91 & 323 \\
2001 & 91 & 64 & 89,69 & 140,14 \\
\hline
\end{tabular}

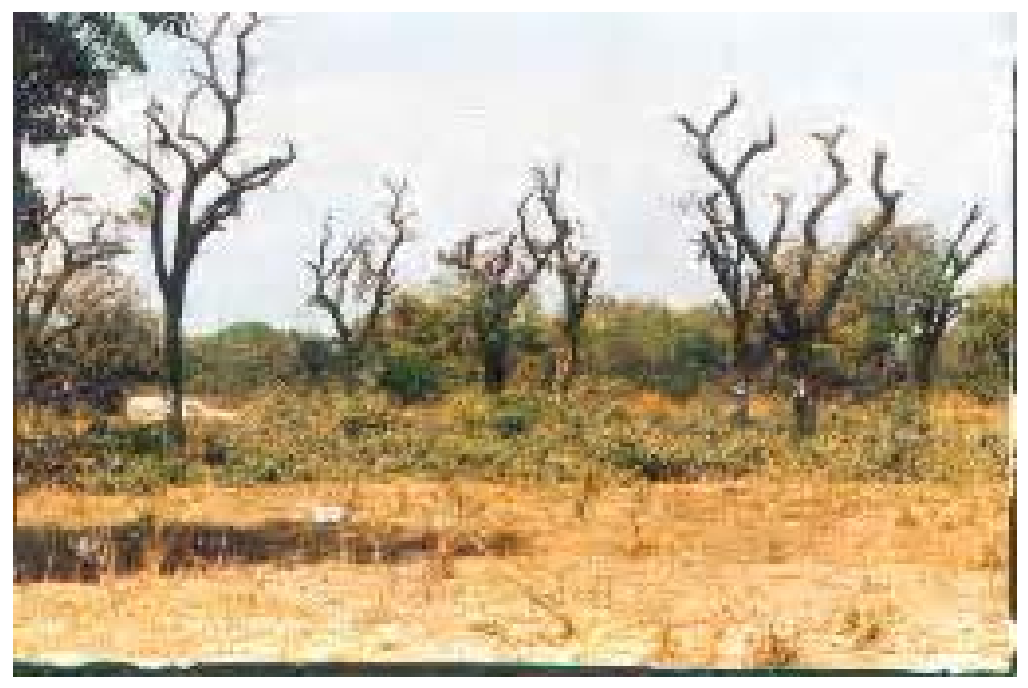

Figure 4 : Arbres de karité émondés (Photo TRAORE, 1997) Pruning shea trees

Tableau 4 : Statistiques élémentaires du degré du parasitisme des karités, après émondage en 1997, dans le parc à karités de Tengrela

Elementary statistics of the degree of parasitism of shea trees, after pruning in 1997, in Tengrela's park of shea trees

\begin{tabular}{ccccc}
\hline \multirow{2}{*}{$\begin{array}{c}\text { Années } \\
\text { d'observation }\end{array}$} & \multicolumn{4}{c}{ Statistique élémentaire } \\
\cline { 2 - 5 } & $\begin{array}{c}\text { Nombre de } \\
\text { karités }\end{array}$ & $\begin{array}{c}\text { Degré de parasitisme } \\
\text { (nombre de parasites/arbre) }\end{array}$ & $\begin{array}{c}\text { Ecart type } \\
\text { (nombre de parasites }\end{array}$ & $\begin{array}{c}\text { Coefficient de } \\
\text { variation (\%) }\end{array}$ \\
\hline 1998 & 91 & 0 & 0 & 0 \\
1999 & 91 & 0,02 & 0,54 & 1050 \\
2000 & 91 & 0,2 & 0,54 & 270 \\
2001 & 91 & 0,24 & 0,24 & 270,83 \\
\hline
\end{tabular}




\section{DISCUSSION}

Le taux élevé de karités parasités (96\%) est très préoccupant. Cette inquiétude est d'autant plus justifiée que même les jeunes karités sont également atteints et que les karités adultes sont susceptibles de porter plusieurs dizaines de parasites. Boussim et al., (1993) trouvent des niveaux d'infestation similaires au Burkina Faso (95\%). Selon ces auteurs, le taux d'infestation peut atteindre $100 \%$ de karités dans certaines localités. La forte variabilité du degré de parasitisme observée suivant les arbres montre que les Loranthaceae ne parasitent pas les karités avec la même fréquence. Existeraient-il des différences de sensibilité des karités aux Loranthaceae ? Des recherches futures trouveront certainement une réponse à la question.

La localisation préférentielle des Loranthaceae à la périphérie du houppier de leur hôte pourrait s'expliquer par un besoin de lumière chez le parasite. En effet, les Loranthaceae sont hémiparasites ; elles puisent les éléments minéraux dans l'hôte grâce à leur suçoir, mais, pour la synthèse des substances organiques, ces parasites usent de leurs propriétés chlorophylliennes. D'où la localisation à la périphérie des houppiers pour recevoir la lumière. De plus, des études botaniques indiquent que les espèces de Loranthaceae d'Afrique ont besoin de la lumière pour germer (Balle et al., 1961). Ainsi, les semences de parasites situées à l'intérieur des houppiers ne recevraient pas assez de lumière pour pouvoir germer.

La variation du degré de parasitisme suivant les parcelles pourrait se comprendre par le système de reproduction et de propagation des Loranthaceae. En effet, selon Boussim et al., (1993), Traoré et al., (1996), la dissémination des Loranthaceae se fait par les oiseaux qui se nourrissent de la viscine (pulpe collante) de leurs fruits. Après consommation de la pulpe, les graines rejetées restent fixées sur une branche de l'arbre hôte. Très souvent, les graines restent collées sur le bec de l'oiseau, par la viscine. Elles sont ainsi transportées et déposées d'arbre en arbre et donnent naissance à de nouveaux plants de parasite. Cela expliquerait le faible niveau de parasitisme sur les arbres des parcelles 6 et 14 où les oiseaux craignent de se poser en raison de la présence humaine. Par contre, la proximité des parcelles 2 et 17 avec le milieu naturel qui favorise leur accès par les oiseaux, expliquerait le fort degré de parasitisme qu'on y a observé.

Selon Boussim et al., (1993), les déformations observées chez l'arbre hôte au niveau des points d'insertion des parasites seraient la conséquence de la réaction des karités à la contamination, tandis que la mort des parasites sur l'hôte pourrait être un phénomène de rejet naturel qu'il conviendrait d'étudier.

Les corrélations négatives observées entre le niveau de parasitisme et la production en fruits du karité, bien que faibles, indiquent cependant que les Loranthaceae influenceraient négativement la production en fruits du karité. Baillon et al., (1987) indiquent effectivement que les Loranthaceae réduisent la production des arbres fruitiers. L'influence négative des Loranthaceae sur la production en fruits, pourrait s'expliquer par le détournement de la sève par les parasites, au détriment des rameaux producteurs. Boussim et al., (1993) ont montré que le parasite une fois fixé sur son hôte, détourne à son profit la sève brute destinée à l'extrémité distale de la branche parasitée qui subit ainsi un déficit trophique entraînant l'arrêt de la croissance et le dépérissement. Les effets cumulés provoquent un affaiblissement généralisé de l'hôte qui explique une floraison et une fructification peu abondantes de celui-ci.

L'absence de fructification des karités, la première année après émondage, serait liée au fait que les arbres consacrent d'abord leurs ressources à la production de rameaux (Hugues, 1987). A partir de 1999, soit 2 ans après émondage, les karités émondés en 1997 entrent en production car, ils ont émis des rameaux capables de fructifier. La grande variabilité observée au niveau de la production en fruits des karités traités serait probablement liée à l'asynchronisme observée dans le développement des karités émondés. En effet, la régénération ne se fait pas avec la même vitesse pour les karités. Si certains arbres émettent rapidement des rameaux fructifères, d'autres par contre tardent à donner des repousses capables de fructifier ; d'où la forte variabilité observée au niveau de la production en fruits. L'évolution rapide de la production en fruits des arbres émondés, montre un effet bénéfique de l'émondage sur le karité. Ceci est encourageant quant à la vulgarisation de l'émondage du karité comme technique culturale permettant de lutter contre les Loranthaceae. 
L'absence de parasites sur les arbres la première année après émondage pourrait s'expliquer d'une part par la qualité de l'opération (tous les parasites ayant été éliminés) et d'autre part par la méfiance des oiseaux propagateurs à se poser sur les arbres traités. En effet, un an après l'émondage, les karités présentent un feuillage peu dense et les branches découvertes ne garantissent pas la sécurité des oiseaux qui, de ce fait, n'osent pas les fréquenter de peur d'être exposés aux rapaces (Soro,1999). A partir de la deuxième année, le feuillage des karités est devenu fréquentable par les oiseaux. D'où l'implantation des parasites sur les plants. Le degré de parasitisme augmente faiblement avec les années, ce qui est encourageant pour l'expérimentation.

\section{CONCLUSION}

Les Loranthaceae, parasites vasculaires longtemps ignorés, constituent une grave menace pour l'environnement et plus particulièrement pour le karité dans les zones soudaniennes. Globalement un peu plus de $96 \%$ des karités sont infestés par ces parasites et subissent de graves dégâts. Beaucoup de karités sont rendus improductifs par ces parasites et meurent à brève échéance.

Le traitement de l'arbre se justifie par les énormes potentialités économiques qu'il présente.

II ressort de cette étude que les zones beaucoup fréquentées par les oiseaux sont les lieux de prédilection des Loranthaceae. Cela est à mettre en relation avec la présence d'oiseaux disséminateurs des graines du parasite où une meilleure sécurité leur est ainsi offerte.

L'émondage apparaît comme une technique culturale permettant de lutter efficacement contre les parasites du karité. II permet de sécuriser et de restaurer la production fruitière de l'arbre qui intervient à partir de la deuxième année.

Une meilleure connaissance de la répartition du parasite, de sa biologie et des dégâts causés sur l'hôte permettrait la mise en place d'un programme de recherche sur la lutte contre les Loranthaceae. Ceci est d'autant plus important que les karités infestés constituent autant de foyers d'infestations potentiels pour les karités encore sains et pour les autres essences.

\section{REFERENCES}

BAILLON (F.) et FROCHOT (H.) 1987. La lutte chimique contre le gui des "feuillus". Pénétration et distribution dans le gui de deux herbicides à absorption foliaire. Phytoma, Défense des cultures, 391 : 16-21.

BALLE (S.) et HALLE (N.). 1961. Les Loranthaceae de la Côte d'Ivoire. Adansonia, nouvelle série, tome $1: 210-265$

BOUSSIM (J.) 1991. Contribution à l'étude des Tapinanthus, parasites du karité au Burkina Faso. Thèse de Doctorat de $3^{\text {éme }}$ cycle, Université de Ouagadougou, 152p.

BOUSSIM et al., 1993. Tapinanthus parasite du karité au Burkina Faso. Bois et Forêts des Tropiques, 238 : 45-63.

DELABRAZE (P.) et LANIER (L.) 1972. Contribution à la lutte chimique contre le gui : Viscum album L. Eur. J. For. Pathol. 2 : 95-103.

FROCHOT (H.), PITCH (M.) et WEHRLEN (L.) 1978 : Différence de sensibilité au Viscum album L. de quelques clones de peuplier Populus $s p$. Bibliothèque nationale, Paris, Compte rendu du $103^{e}$ congrès national des Sociétés Savantes, Nancy-Metz. Tome 1. 157-165.

HUGUES (D.) 1987. jardins et vergers d'Afrique. Belgique : Terre et vie, Paris : l'Harmattan, Douala APICA, Pays-Bas : CTA, Dakar: ENDA. 354 p.

SALLE (G.), (I. J.) BOUSSIM, (A) RAYNAL-ROQUES et (F.) BRUNCK. 1991. Le karité : Une richesse potentielle. Perspectives de recherches pour améliorer sa production (Revue bois et forêts des tropiques). $\mathrm{N}^{\circ} 228$, $2^{\mathrm{è}}$ trimestre : 1-23.

SORO, D. (1999). Déparasitage par émondage et production en fruits du parc naturel à karités de Tengrela, dans le Nord de la Côte d'Ivoire. Mémoire de D.E.A., Laboratoire de Botanique, U.F.R Biosciences, Université de Cocody, Côte d'Ivoire, $80 \mathrm{p}$.

TRAORE (D.) et DA (K. P.). 1996. Lutte contre les plantes vasculaires parasites du karité et du néré dans le Nord de la Côte d'lvoire. Cas des Départements de Korhogo, Boundiali, Ferkessedougou et Tengrela. Rapport annuel de PEP A.I.S.A-Cl., 97 p.

TRAORE(D.) et DA (K. P.). 1997. Lutte contre les plantes vasculaires parasites du karité et du néré dans le Nord de la Côte d'Ivoire. Cas des Départements de Korhogo, Boundiali, Ferkessedougou et Tengrela. Rapport annuel de PEP A.I.S.A-Cl., 16 p. 